\title{
Using Combinatorial Optimization Methods for Quantification Scheduling ${ }^{\star}$
}

\author{
P. Chauhan ${ }^{1}$, E. Clarke ${ }^{1}$, S. Jha ${ }^{2}$, J. Kukula ${ }^{3}$, H. Veith ${ }^{4}$, and D. Wang ${ }^{1}$ \\ 1 Carnegie Mellon University \\ 2 University of Wisconsin - Madison \\ 3 Synopsys Inc. \\ 4 TU Vienna, Austria
}

\begin{abstract}
Model checking is the process of verifying whether a model of a concurrent system satisfies a specified temporal property. Symbolic algorithms based on Binary Decision Diagrams (BDDs) have significantly increased the size of the models that can be verified. The main problem in symbolic model checking is the image computation problem, i.e., efficiently computing the successors or predecessors of a set of states. This paper is an in-depth study of the image computation problem. We analyze and evaluate several new heuristics, metrics, and algorithms for this problem. The algorithms use combinatorial optimization techniques such as hill climbing, simulated annealing, and ordering by recursive partitioning to obtain better results than was previously the case. Theoretical analysis and systematic experimentation are used to evaluate the algorithms.
\end{abstract}

\section{Introduction}

Model Checking and State Explosion. In model checking [CGP00], the system to be verified is represented as a finite Kripke structure or labelled transition system. A Kripke structure over a set of atomic propositions $A P$ is a tuple $K=(S, R, L, I)$ where $S$ is the set of states, $R \subseteq S \times S$ is the set of transitions, $I \subseteq S$ is the non-empty set of initial states, and $L: S \rightarrow 2^{A P}$ labels each state by a set of atomic propositions.

Given a Kripke structure $K=(S, R, I, L)$ and a specification $\phi$ in a temporal logic such as CTL, the model checking problem is the problem of finding all states $s$ such that $K, s \models \phi$ and checking if the initial states are among these. Model checking algorithms usually exploit the fact that temporal operators can

* This research is sponsored by the Semiconductor Research Corporation (SRC), the Gigascale Research Center (GSRC), the National Science Foundation (NSF) under Grant No. CCR-9505472, and the Max Kade Foundation. One of the authors is also supported by Austrian Science Fund Project N Z29-INF. Any opinions, findings and conclusions or recommendations expressed in this material are those of the authors and do not necessarily reflect the views of GSRC, NSF, or the United States Government. 
be characterized as $\mu$-calculus terms. For example, the set of states where the CTL formula $\mathbf{E F} \phi$ holds, is given by

$$
\mathbf{E F} \phi \equiv \mu S . \phi \vee \mathbf{E X} S .
$$

Recall that $\mathbf{E F} \phi$ expresses reachability, i.e., the existence of a path where $\phi$ eventually holds. Such fixpoint translations directly correspond to iterative algorithms.

Symbolic Verification. In practice, the systems to be verified are described by programs in finite state languages such as SMV or VERILOG. These programs are then compiled into equivalent Kripke structures. The main practical problem in model checking is the state explosion problem: the size of the state space of the system is exponential in the size of its description. Therefore, even for systems of relatively modest size, it is often impossible to compute their state space explicitly.

In symbolic verification, the transition relation of the Kripke structure is not explicitly constructed, but instead a Boolean function representing the transition relation is computed. Sets of states are also represented as Boolean functions. Then, the fixpoint algorithms are applied to the formulas rather than to the Kripke structure. Since the Boolean formula is usually exponentially smaller than an explicit representation, symbolic verification is often able to alleviate the state explosion problem in these situations. Binary Decision Diagrams (BDDs) have been a particularly useful data structure for representing Boolean functions; in addition to their relative succinctness they provide a canonical representation for Boolean functions. As a result, equality of two Boolean functions can be easily checked in this representation.

At the core of all symbolic algorithms is image computation, ${ }^{1}$ i.e., the task of computing the set of successors $\operatorname{Img}(S)$ of a set of states $S$, where

$$
\operatorname{Img}(S):=\left\{s^{\prime}: \exists s \cdot R\left(s, s^{\prime}\right) \wedge s \in S\right\} .
$$

Image computation is one of the major bottlenecks in verification. Often it is impossible to construct a single $\mathrm{BDD}$ for the transition relation $R$. Instead, $R$ is represented as a partitioned transition relation, i.e., as the conjunction of several BDDs, each representing a piece of $R$. The problem is how to compute $\operatorname{Img}(S)$ without actually computing $R$.

As the above definition of Img indicates, the process of image computation involves quantifying state variables. In the BDD representation, this amounts to quantifying over several Boolean state variables. Early quantification [BCL91b, $\left.\mathrm{TSL}^{+} 90\right]$ is a technique which attempts to reorder the conjuncts so that the scope of each quantifier is minimized. The effect of early quantification is that the evaluation of single quantifiers can be done over relatively small intermediate $B D D s$. An exact definition of early quantification will be given in Sect. 2. The

\footnotetext{
${ }^{1}$ The techniques in this paper also apply to preimage computation. For ease of exposition, we restrict ourselves to image computation.
} 
success of early quantification hinges heavily upon the derivation and ordering of sub-relations. Significant effort has been directed over the last decade to this problem. Since the problem is known to be NP-hard, various heuristics have been proposed for the problem.

In this paper, we propose and analyze several new techniques for efficient image computation using the partitioned representation. The main contributions of the paper are the following:

- We extend and analyze image computation techniques previously developed by Moon et al. [MKRS00]. These techniques are based on the dependence matrix of the partitioned transition relation. We explore various lifetime metrics related to this representation and argue their importance in predicting costs of image computation. Moreover, we provide effective heuristic techniques to optimize these metrics.

- We show that the problem of minimizing the lifetime metric of [MKRS00] is NP complete. More importantly, the reduction used to prove this result explains the close connection between efficient image computation and the well studied problem of computing the optimal linear arrangement for an undirected graph. - We model the interaction between various sub-relations in the partitioned transition relation as a weighted graph, and introduce a new class of heuristics called ordering by recursive partitioning.

- We have performed extensive experiments which indicate the effectiveness of our techniques. By implementing these techniques, we have also contributed to the code base of the symbolic model checker NuSMV [CCGR99].

The main conclusion to be drawn from our analysis is the following: For complicated industrial designs, the effort initially spent on ordering algorithms is clearly amortized during image computation. In other words, the benefits of good orderings outweigh the cost of slow combinatorial optimization algorithms.

The remainder of this paper is organized as follows: in Sect. 2, we introduce notations and definitions used throughout the paper. Section 3 reviews the state of the art for this problem. Section 4 discusses various algorithms that facilitate early quantification. Section 5 describes experimental results. Finally, we conclude in Sect. 6 with some directions for future research.

\section{Preliminaries}

Notation: Every state is represented as a vector $b_{1} \ldots b_{n} \in\{0,1\}^{n}$ of Boolean values. The transition relation $R$ is represented by a Boolean function $T\left(x_{1}, \ldots, x_{n}\right.$, $\left.x_{1}^{\prime}, \ldots, x_{n}^{\prime}\right)$. Variables $X=x_{1}, x_{2}, \ldots, x_{n}$ and $X^{\prime}=x_{1}^{\prime}, x_{2}^{\prime}, \ldots, x_{n}^{\prime}$ are called current state and next state variables respectively. $T\left(X, X^{\prime}\right)$ is an abbreviation for $T\left(x_{1}, \ldots, x_{n}, x_{1}^{\prime}, \ldots, x_{n}^{\prime}\right)$. Similarly, functions of the form $S(X)=S\left(x_{1}, \ldots, x_{n}\right)$ describe sets of states. We will occasionally refer to $S$ as the set, and to $T$ as the transition relation. For simplicity we will use $X$ to denote both the set $\left\{x_{1}, \ldots, x_{n}\right\}$ and the vector $\left\langle x_{1}, \ldots, x_{n}\right\rangle$. Then the set of variables on which $f$ depends is denoted by $\operatorname{Supp}(f)$. 
Example 1. [3 bit counter. (Running Example)] Consider a 3-bit counter with bits $x_{1}, x_{2}$ and $x_{3} . x_{1}$ is the least significant and $x_{3}$ the most significant bit. The state variables are $X=x_{1}, x_{2}, x_{3}, X^{\prime}=x_{1}^{\prime}, x_{2}^{\prime}, x_{3}^{\prime}$. The transition relation of the counter can be expressed as

$$
T\left(X, X^{\prime}\right)=\left(x_{1}^{\prime} \leftrightarrow \neg x_{1}\right) \wedge\left(x_{2}^{\prime} \leftrightarrow x_{1} \oplus x_{2}\right) \wedge\left(x_{3}^{\prime} \leftrightarrow\left(x_{1} \wedge x_{2}\right) \oplus x_{3}\right) .
$$

In later examples, we will compute the image $\operatorname{Img}(S)$ of the set $S(X)=\neg x_{1}$. Note that $S(X)$ contains those states where the counter is even.

Partitioned BDDs: For most realistic designs it is impossible to build a single $\mathrm{BDD}$ for the entire transition relation. Therefore, it is common to represent the transition relation as a conjunction of smaller BDDs $T_{1}\left(X, X^{\prime}\right), T_{2}\left(X, X^{\prime}\right), \ldots$, $T_{l}\left(X, X^{\prime}\right)$, i.e.,

$$
T\left(X, X^{\prime}\right)=\bigwedge_{1 \leq i \leq l} T_{i}\left(X, X^{\prime}\right)
$$

where each $T_{i}$ is represented as a BDD. The sequence $T_{1}, \ldots, T_{l}$ is called a partitioned transition relation. Note that $T$ is not actually computed, but only the $T_{i}$ 's are kept in memory.

Example 2. [3 bit counter, ctd.] For the 3 bit counter, a very simple partitioned transition relation is given by the functions $T_{1}=\left(x_{1}^{\prime} \leftrightarrow \neg x_{1}\right), T_{2}=\left(x_{2}^{\prime} \leftrightarrow\right.$ $\left.x_{1} \oplus x_{2}\right)$ and $T_{3}=\left(x_{3}^{\prime} \leftrightarrow\left(x_{1} \wedge x_{2}\right) \oplus x_{3}\right)$.

Partitioned transition relations appear naturally in hardware circuits where each latch (i.e., state variable) has a separate transition function. However, a partitioned transition relation of this form typically leads to a very large number of conjuncts. A large partitioned transition relation is similar to a CNF representation. So as the number of conjuncts increases, the advantages of BDDs are gradually lost. Therefore, starting with a very fine partition $T_{1}, \ldots, T_{l}$ obtained from the bit relations, the conjuncts $T_{i}$ are grouped together into clusters $C_{1}, \ldots, C_{r}, r<l$ such that each $C_{i}$ is a BDD representing the conjunction of several $T_{i}$ 's. The image $\operatorname{Img}(S)$ of $S$ is given by the following expression.

$$
\begin{aligned}
\operatorname{Img}(S(X)) & =\exists X \cdot\left(T\left(X, X^{\prime}\right) \wedge S(X)\right) \\
& =\exists X \cdot\left(\bigwedge_{1 \leq i \leq l} T_{i}\left(X, X^{\prime}\right) \wedge S(X)\right) \\
& =\exists X \cdot\left(\bigwedge_{1 \leq i \leq r} C_{i}\left(X, X^{\prime}\right) \wedge S(X)\right)
\end{aligned}
$$

Note that in general $\exists x(\alpha \wedge \beta)$ is not equivalent to $(\exists x \alpha) \wedge(\exists x \beta)$. Consequently, to compute $\operatorname{Img}(S(X))$, formula 3 instructs us to compute first a BDD for $\bigwedge_{1 \leq i \leq r} C_{i}\left(X, X^{\prime}\right) \wedge S(X)$. As argued above, partitioned transition relations have been introduced to avoid computing this potentially large BDD. 
Early Quantification: Under certain circumstances, existential quantification can be distributed over conjunction using early quantification [BCL91b, TSL ${ }^{+}$90]. Early quantification is based on the following observation: if we know that $\alpha$ does not contain $x$, then $\exists x(\alpha \wedge \beta)$ is equivalent to $\alpha \wedge(\exists x \beta)$. In general, we have $l$ conjuncts and $n$ variables to be quantified. Since loosely speaking, clusters correspond to semantic entities of the design to be verified, it is expected that not all variables appear in all clusters. Therefore, some of the quantifications may be shifted over several $C_{i}$ 's. For a given sequence $C_{1}, \ldots, C_{r}$ of clusters, we obtain

$$
\begin{gathered}
\operatorname{Img}(S(X))=\exists X_{1} \cdot\left(C _ { 1 } ( X , X ^ { \prime } ) \wedge \exists X _ { 2 } \cdot \left(C_{2}\left(X, X^{\prime}\right) \ldots\right.\right. \\
\left.\left.\exists X_{r} \cdot\left(C_{r}\left(X, X^{\prime}\right) \wedge S(X)\right)\right)\right)
\end{gathered}
$$

where $X_{i}$ is the set of variables which do not appear in $\operatorname{Supp}\left(C_{1}\right) \cup \ldots \cup$ $\operatorname{Supp}\left(C_{i-1}\right)$ and each $X_{i}$ is disjoint from each other. Existentially quantifying out a variable from a formula $f$ reduces $|S u p p(f)|$ which usually corresponds to a reduced BDD size. The success of early quantification strongly depends on the order of the conjuncts $C_{1}, \ldots, C_{r}$.

Quantification Scheduling. The size of the intermediate BDDs in image computation can be reduced by addressing the following two questions:

Clustering: How to derive the clusters $C_{1}, \ldots, C_{r}$ from the bit-relations $T_{1}, \ldots, T_{l}$ ?

Ordering: How to order the clusters so as to minimize the size of the intermediate BDDs?

These two questions are not independent. In particular, a bad clustering results in a bad ordering. Moon and Somenzi [MS00] refer to this combined problem as the quantification scheduling problem. The ordering of clusters is known as the conjunction schedule.

Our algorithms are based on the concepts of dependence matrices (introduced in [MKRS00,MS00]) and sharing graphs.

Definition 1 (Moon et al). The dependence matrix of an ordered set of functions $\left\{f_{1}, f_{2}, \ldots, f_{m}\right\}$ depending on variables $x_{1}, \ldots, x_{n}$ is a matrix $D$ with $m$ rows and $n$ columns such that $d_{i j}=1$ if function $f_{i}$ depends on variable $x_{j}$, and $d_{i j}=0$ otherwise.

Thus, each row corresponds to a formula, and each column to a variable. For image computation, we will associate the rows with the conjuncts of the partitioned transition relation, and the columns with the state variables. For example, $f_{m}=S(X), f_{m-1}=C_{r}, \ldots$. Thus, different choices for $f_{i}, 1 \leq i \leq m$ correspond to different orderings.

We will assume that the conjunction is taken in the order $f_{m}, f_{m-1}, \ldots, f_{2}, f_{1}$, i.e., we consider an expression of the form $\exists X\left(f_{1} \wedge\left(f_{2} \wedge \ldots \wedge\left(f_{m-1} \wedge f_{m}\right)\right)\right)$. If a variable occurs only in $f_{m}$, we can quantify it early by pushing it to the right just before $f_{m}$. 
Example 3. [3 bit counter, ctd.] For $f_{4}=S(X), f_{3}=T_{3}, f_{2}=T_{2}, f_{1}=T_{1}$ the dependency matrix for our running example looks as follows:

\begin{tabular}{r|cccccc} 
& $v_{1}$ & $v_{2}$ & $v_{3}$ & $v_{1}^{\prime}$ & $v_{2}^{\prime}$ & $v_{3}^{\prime}$ \\
\hline$f_{1}=T_{1}$ & 1 & 0 & 0 & 1 & 0 & 0 \\
$f_{2}=T_{2}$ & 1 & 1 & 0 & 0 & 1 & 0 \\
$f_{3}=T_{3}$ & 1 & 1 & 1 & 0 & 0 & 1 \\
$f_{4}=S(X)$ & 1 & 0 & 0 & 0 & 0 & 0
\end{tabular}

In general, for a variable $x_{j}$, let $l_{j}$ denote the smallest index $i$ in column $j$ such that $d_{i j}=1$. Analogously, $h_{j}$ denotes the largest index. We can quantify away the variable $x_{j}$ as soon as the conjunct corresponding to the row $l_{j}$ has been considered. The variable does not appear in any conjuncts after $h_{j}$. Hence, $h_{j}-l_{j}$ can be viewed as the lifetime of a variable. Moon, Kukula, Ravi and Somenzi [MKRS00] define the following metric and use it extensively in their algorithms.

Definition 2 (Moon, Kukula, Ravi, Somenzi). The normalized average lifetime of the variables in a dependence matrix $D_{m \times n}$ is given by

$$
\lambda=\frac{\sum_{1 \leq j \leq n}\left(h_{j}-l_{j}+1\right)}{m \cdot n}
$$

Note that the definition of $\lambda$ assumes that $S(X)$ is given. Therefore, since $\lambda$ depends on $S(X)$, the ordering has to be recomputed in each step of the fixpoint computation. We are considering static ordering techniques here, which are computed independently of any particular $S(X)$, so it is necessary to make assumptions about the structure of $S(X)$. We obtain two lifetime metrics $\lambda_{U}$ and $\lambda_{L}$ depending on whether we assume $\operatorname{Supp}(S)=X$ or $\operatorname{Supp}(S)=\emptyset$. It is easy to see that $\lambda_{L} \leq \lambda \leq \lambda_{U}$. The terms average active lifetime and total active lifetime are also used to denote $\lambda_{L}$ and $\lambda_{U}$ respectively. Moon and Somenzi argue in favour of using $\lambda_{L}$. We will evaluate the effectiveness of each of these metrics to predict image computation costs.

\section{Related Work}

The importance of the clustering and ordering problem was first recognized by Burch et al. [BCL91a] and Touati et al. [TSL+90]. Geist and Beer [GB94] proposed a simple heuristic algorithm, in which they ordered conjuncts in the increasing order of the number of support variables. All these techniques are static techniques. Subsequently, the same clusters and ordering are used for all the image computations during symbolic analysis. Since the clustering and ordering problems are not independent, these techniques typically begin by first ordering the conjuncts and then clustering them and finally ordering the clusters again using the same heuristics. The first successful heuristic (commonly known as IWLS95) for this problem is due to Ranjan et al. [RAP $\left.{ }^{+} 95\right]$. They have an elaborate heuristic procedure for ordering the initial conjuncts and the clusters. 
The ordering procedure maintains a set $Q$ of conjuncts that are already ordered and a set $R$ of conjuncts that are yet to be ordered. Note that we have used the word conjunct here to mean both the conjuncts before clustering and the clusters in the final ordering phase. The next conjunct in the order is chosen from $R$ using a heuristic score. The score is computed by using four factors: the maximum BDD index of a variable that can be quantified, the number of next state variables that would be introduced, the number of support variables, and the number of variables that will be quantified away. After the ordering phase, the clusters are derived by repeatedly conjoining the conjuncts until the BDD of the cluster grows larger than some partition size limit, at which point a new cluster is started. Bwolen Yang proposed a similar technique in his thesis [Yan99]. However, he introduces a pre-merging phase where conjuncts are initially merged pairwise based on the sharing of support variables and the maximum BDD size constraint. His ordering heuristic is based on six factors which are similar to those used by Ranjan et al. $\left[\mathrm{RAP}^{+} 95\right]$. However, he also takes into account the relative growth in BDD sizes. The clustering algorithm Yang uses is the same as the one used in IWLS95. A recent paper by Moon and Somenzi [MS00] presents an ordering algorithm (henceforth referred to as FMCAD00) based on computing the Bordered Block Triangular form of the dependence matrix. Their clustering algorithm is based on the sharing of support variables (affinity). They report large performance gains with respect to the IWLS95 technique.

\section{Algorithms for Ordering Clusters}

The algorithms we propose also follow the order-cluster-order strategy. The ordering algorithms that we present in this section are used before and after clustering. Our clustering strategy is as in IWLS95. For the sake of clarity of notation, let us assume that the clusters $C_{1}, C_{2}, \ldots, C_{r}$ have been constructed and we are ordering them. But the discussion applies equally well to ordering the initial conjuncts $T_{1}, \ldots, T_{n}$.

We present two classes of algorithms. The first one is based on dependence matrix and the other one on sharing graphs.

In Sect. 2 we defined a dependence matrix $D$ corresponding to the set of clusters $C_{1}, \cdots, C_{r}$. As already pointed out, the number of support variables provides a good estimate of the size of a BDD. Therefore, we seek a schedule in which the lifetime of variables is low. Moon and Somenzi [MS00] provide a method to convert a dependence matrix into bordered block triangular form with the goal of reducing $\lambda_{L}$.

\subsection{Minimizing $\lambda$ is NP-Complete}

The main result of this subsection (Theorem 1) motivates the use of various combinatorial optimization methods.

Let $\lambda$-OPT be the following decision problem: given a dependence matrix $D$ and a number $r$, does there exist a permutation $\sigma$ of the rows of $D$ such 
that $\lambda<r$ ? The following theorem shows that $\lambda-O P T$ is NP-complete. The reduction is from the optimal linear arrangement problem (OLA) [GJ79, page 200]. Due to space limitations the proof is given in the appendix.

Theorem 1. $\lambda$-OPT is NP-complete.

The complexity of this problem was not explored by Moon and Somenzi [MS00]. There exists a variety of heuristics for solving the optimal linear arrangement problem and related problems in combinatorial optimization. Some of these heuristics are based on hill climbing and simulated annealing. There are two important characteristics of this class of algorithms. First of all, they all try to minimize an underlying cost function. Second, these heuristics use a finite set of primitive transformations, which allows them to move from one solution to another. In our case, the set of swaps of the rows of the dependence matrix constitutes the set of moves and the cost function can be chosen to be either $\lambda_{L}$ or $\lambda_{U}$. Our experimental results (Sect. 5) confirm that $\lambda_{L}$ correlates with image computation costs much better than $\lambda_{U}$ does, in accordance with the claim of [MS00]. Simulated annealing is a more general and flexible strategy than hill climbing.

\subsection{Hill Climbing}

Hill climbing is the simplest greedy strategy in which at each point, the solution is improved by choosing two rows to be swapped in such a manner as to achieve best improvement in the cost function. This process is repeated until no further move improves the solution. Since the best move is chosen at each point, this strategy is also called steepest descent hill climbing. However, this algorithm can easily get stuck in local optima. Randomization is used to alleviate this problem as follows: The best move that improves the solution is accepted only with some probability $p$, and with probability $1-p$, a random move is accepted. This allows the algorithms to get out of local optima. Note that with $p=1.0$, we get the steepest descent hill climbing. The algorithm can be run multiple number of times, each time beginning with a random permutation, and the best solution that is achieved is accepted.

Figure 1 describes the algorithm in exact terms. The hill climbing procedure is repeated NumStarts times. In the algorithm, $\sigma$ denotes a permutation of the rows of the dependency matrix. Hill climbing is performed until no further improvement in $\lambda$ is possible.

\subsection{Simulated Annealing}

The physical process of annealing involves heating a piece of metal and letting it cool down slowly to relieve stresses in the metal. The simulated annealing algorithm (introduced by Metropolis et al. $\left[\mathrm{MRR}^{+} 53\right]$ ) mimicks this process to solve large combinatorial optimization problems [KJV83]. Drawing analogy from the physical process of annealing, the algorithm begins at a high "temperature", 


\section{$\operatorname{HillClimbOrder}(D)$}

$1 \quad \lambda_{\text {best }}=2 / /$ any number greater than 1 will do, since $\lambda$ is always less than 1

2 for $i=1$ to NumStarts

3 let $\sigma^{\prime}$ be a random permutation of conjuncts.

\section{while there exists a swap in $\sigma^{\prime}$ to reduce $\lambda$}

make the best swap with probability $p$,

or make a random swap with probability $1-p$ to update $\sigma^{\prime}$. if $\lambda^{\prime}<\lambda_{\text {best }}$

$$
\begin{aligned}
& \lambda_{\text {best }}=\lambda^{\prime} \\
& \sigma_{\text {best }}=\sigma
\end{aligned}
$$

endif

\section{endfor}

Fig. 1. Hill climbing algorithm for minimizing $\lambda$

where the set of moves is essentially random. This allows larger jumps from local to global optima. Gradually, the temperature is decreased and the moves become less random favoring greedy moves over random moves for achieving a global optimum. Finally, the algorithm terminates at "freezing" temperatures where no further moves are possible. At each stage, the temperature is kept constant until "thermal quasi-equilibrium" is reached. While random moves help in the beginning, when the algorithm has a greater tendency to get stuck in local optima, the greedy moves help to achieve a global optimum once the solution is in the proximity of one. In practice, simulated annealing has been successfully used to solve optimization problems from several domains.

The probability of making a move that increases the cost function is related to the temperature $t_{i}$ at the $i$-th iteration, and is given by $e^{-\Delta \lambda / t_{i}}$. Thus at higher temperatures, the probability of accepting random moves is high. The gradual decrease of temperature is called the cooling schedule. If the temperature is decreased by a fraction $r$ in each stage, we get an exponential cooling schedule. Thus beginning with an initial temperature of $t_{0}$, the temperature in the $i$ th iteration is $t_{0} r^{i}$. It has been shown that a logarithmic cooling schedule is guaranteed to achieve an optimal solution with high probability [B'e92,Haj85]. However, this is an extremely slow cooling schedule and simple cooling schedules like exponential schedules perform well for many problems. Figure 2 describes our algorithm. The parameter NumStarts controls the number of times the temperature is decreased. The parameter NumStarts 2 controls the number of iterations at a fixed temperature $t_{i}$.

\subsection{Sharing Graphs and Separators}

We build sharing graphs as defined below to model interaction between clusters. 


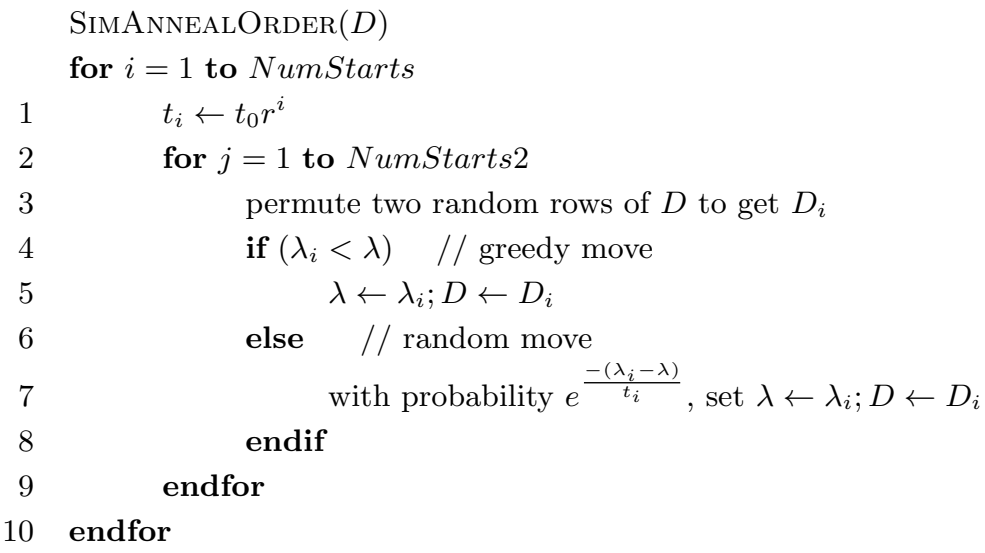

Fig. 2. Simulated annealing algorithm to minimize $\lambda$

Definition 3. A sharing graph corresponding to a set of Boolean functions $\left\{f_{1}, f_{2}, \ldots, f_{m}\right\}$ is a weighted graph $G\left(V, E, w_{e}\right)$, where $V=\left\{f_{1}, f_{2}, \ldots, f_{m}\right\}$, $E=V \times V$ and $w_{e}: E \rightarrow \Re$ is a real-valued weight function.

We shall use heuristic weight functions to express interaction between clusters. Intuitively, the stronger the interaction between two clusters, the closer they should be in the ordering. IWLS95 and Bwolen Yang's heuristics order the conjuncts based on this type of interaction between conjuncts. We propose to use graph algorithms on sharing graphs to order the conjuncts. We define the weight $w\left(T_{i}, T_{j}\right)$ of an edge $\left(T_{i}, T_{j}\right)$ in the sharing graph as

$$
w\left(T_{i}, T_{j}\right)=W_{1} \cdot \frac{\operatorname{Supp}\left(T_{i}\right) \cap \operatorname{Supp}\left(T_{j}\right)}{\left|\operatorname{Supp}\left(T_{i}\right)\right|+\left|\operatorname{Supp}\left(T_{j}\right)\right|}+W_{2} \cdot \frac{\operatorname{BddSize}\left(T_{i} \wedge T_{j}\right)}{\operatorname{BddSize}\left(T_{i}\right)+\operatorname{BddSize}\left(T_{j}\right)}
$$

The first factor $\left(W_{1} \geq 0\right)$ denotes the relative weight of sharing of support between two conjuncts, while the second factor $\left(W_{2} \leq 0\right)$ denotes the weight of the relative growth in the sizes of BDDs if these two conjuncts are conjoined. Therefore, a higher edge weight between two conjuncts indicates a higher degree of interaction and consequently these conjuncts should appear "close" in the ordering.

A separator partitions the vertices of a weighted undirected graph into two sets such that the total weight of the edges between two partitions is "small". Formally, an edge separator is defined as follows:

Definition 4. Given a weighted undirected graph $G(V, E)$ with two weight functions $w_{e}: E \rightarrow \Re$ and $w_{v}: V \rightarrow \Re$, and a positive constant $\gamma<0.5$, an edge separator is a collection of edges $E_{s}$ such that removing $E_{s}$ from $G$ partitions $G$ into two disconnected subgraphs $V_{1}$ and $V_{2}$, and $\frac{\left|\sum_{v \in V 1} w_{v}(v)-\sum_{v \in V 2} w_{v}(v)\right|}{\sum_{v \in V} w_{v}(v)}<\gamma$. 
Usually, $\gamma$ is chosen very close to zero so that the size of the two sets is approximately the same. The weight of the edge separator $E_{s}$ is simply the sum of the weight of the edges in $E_{s}$. It has been shown that finding an edge separator of minimum weight is NP-complete [GJ79, pp. 209], in fact finding an approximation is NP-hard, too [BJ92]. The problem of finding a good separator occurs in many different contexts and a wide range of application areas. A large number of heuristics have been proposed for the problem. One of the most important heuristics is due to Kernighan and Lin [KL70]. Variations of this heuristic [FM82] have been found to work very well in practice.

By finding a good edge separator of the sharing graph, we obtain two sets of vertices with a low level of interaction between them. Thus the vertices of these two sets can be put apart in the ordering. A complete ordering is achieved by recursively invoking the algorithm on the two halves. Since this ordering respects the interaction strengths between conjuncts, we expect to achieve smaller BDD sizes.

We use the Kernighan-Lin algorithm for finding a good edge separator $E_{s}$. This produces two sets of vertices $L$ and $R$. A vertex $v \in L$ that has an edge of non-zero weight to a vertex in $R$ is called an interface vertex. $L_{I}$ denotes the set of interface vertices in $L$. Similarly, $R_{I}$ denotes the set of interface vertices in $R$. We invoke the algorithm to recursively order $L \backslash L_{I}, L_{I}, R_{I}$, and $R \backslash R_{I}$. Finally, the order on the vertices is given by the order on $L \backslash L_{I}$ followed by the order on $L_{I}$, followed by the order on $R_{I}$, and followed by the order on $R \backslash R_{I}$. Figure 3 describes the complete algorithm.

$\operatorname{KLinOrder}(G(V, E), W)$

1 Find a separator $E_{s}$ using

Kernighan-Lin heuristic

2 Let $L$ and $R$ be two partitions of vertices induced by $E_{s}$.

$3 \quad L_{i} \leftarrow$ Interface $(L)$.

$4 \quad R_{i} \leftarrow$ Interface $(R)$.

5 Recursively call the procedure on the subgraphs induced by $L \backslash L_{i}, L_{i}$, $R_{i}$ and $R \backslash R_{i}$.

6 Order the vertices as $K L i n O r d e r\left(L \backslash L_{i}\right) \prec K L i n O r d e r\left(L_{i}\right) \prec$ $K L i n O r d e r\left(R_{i}\right) \prec K L i n O r d e r\left(R \backslash R_{i}\right)$.

Fig. 3. An ordering algorithm based on graph separators

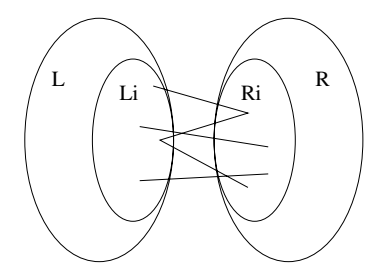

Fig. 4. Kernighan-Lin partition 


\section{$5 \quad$ Experimental Results}

In order to evaluate the effectiveness of our algorithms, we ran reachability and model checking experiments on circuits obtained from the public domain and industry. The "S" series of circuits are ISCAS'93 benchmarks, and the "IU" series of circuits are various abstractions of an interface control circuit from Synopsys. For a fair comparison, we implemented all the techniques in the NuSMV model checker. All experiments were done on a $200 \mathrm{MHz}$ quad Pentium Pro processor machine running the Linux operating system with 1GB of main memory. We restricted the memory usage to $900 \mathrm{MB}$, but did not set a time limit. The two performance metrics we measured are running time and peak number of live $B D D$ nodes. We provided a prior ordering to the model checker and turned off the dynamic variable reordering option. This was done so that the effects of BDD variable reordering do not "pollute" the result. We also recorded the fraction of time spent in the clustering and ordering phases. The cost of these phases is amortized over several image computations performed during model checking and reachability analysis.

In the techniques that we have described, several parameters have to be chosen. For example, the cooling schedule in the case of simulated annealing needs to be determined. We ran extensive "tuning experiments" to find the best value for these parameters. Due to space constraints, we do not describe all those experiments. However, the choice of lifetime metric to optimize is a crucial one and hence in our first set of experiments, we evaluate the effectiveness of these metrics for predicting image computation costs.

Our algorithms for combinatorial optimization of lifetime metrics can choose to work with either upper or lower approximations of lifetimes. We ran the following experiment to estimate the correlation between the performance, and $\lambda_{L}$ and $\lambda_{L}$ respectively. We generate various conjunction schedules for a number of benchmarks by different ordering methods and by varying various parameters of the optimization methods. Each schedule gives us different values for lifetime metrics. We measure the running time and the peak number of live BDD nodes used for the model checking or reachability phase. For each circuit, this gives us four scatter plots for running time vs lifetime metric and space vs lifetime metric. A statistical correlation coefficient between runtime/space and lifetime metric indicates the effectiveness of a metric for predicting the runtime/space requirement. The following Table 1 concisely summarizes the correlation results.

It is clear from this data that the active lifetime $\left(\lambda_{L}\right)$ is a much more accurate predictor of image computation costs than total lifetime $\left(\lambda_{U}\right)$. Hence, simulated annealing and hill climbing techniques optimize $\lambda_{L}$.

In the following set of experiments (Table 2), we compare our techniques against the FMCAD00 strategy [MS00]. The first column indicates the total running time of the benchmark (including ordering/clustering and model checking/reachability phases), the second column indicates the peak number of live BDD nodes in thousands during the whole computation, the third column indicates time used by ordering phase, the next two columns indicate $\lambda_{L}$ and $\lambda_{U}$ achieved. From hill climbing and simulated annealing, we only report the results 
Table 1. Correlation between various lifetime metrics and runtime/space for a representative sample of benchmarks

\begin{tabular}{||l||r|r||r|r||}
\hline \multirow{2}{*}{ Circuit } & \multicolumn{2}{c||}{ Runtime } & \multicolumn{2}{c||}{ Space } \\
\cline { 2 - 5 } & $\lambda_{L}$ & $\lambda_{U}$ & $\lambda_{L}$ & $\lambda_{U}$ \\
\hline \hline IUU0 & 0.560 & 0.303 & 0.610 & 0.227 \\
\hline IU70 & 0.603 & 0.336 & 0.644 & 0.263 \\
\hline TCAS & 0.587 & 0.366 & 0.628 & 0.240 \\
\hline S1269 & 0.536 & 0.402 & 0.559 & 0.345 \\
\hline S3271 & 0.572 & 0.350 & 0.602 & 0.297 \\
\hline
\end{tabular}

of simulated annealing, as both of them belong to the same class of algorithms. Moreover, we found out that in general, simulated annealing achieves better performance than hill climbing.

The algorithm KLin based on edge separators achieves lower peak live node count for several circuits than FMCAD00. For the 15 large benchmarks for which FMCAD'00 takes more than 100 secs to finish, KLin wins 10 cases in terms of Peak live BDD nodes, and 7 cases in terms of running time. In some cases, the savings in space is $40 \%$.

The result for the simulated annealing algorithm that minimizes $\lambda$ is shown in Table 2. Again, in comparison to FMCAD00, for the 15 non-trivial benchmarks, simulated annealing wins 14 cases and ties for the other in space, and wins 11 cases in time. In some cases, the savings in space is $55 \%$. The simulated annealing algorithm can also complete 16 reachability steps for the S1423 circuit, which to our knowledge has not be achieved by other techniques. Comparing KLin and simulated annealing, simulated annealing achieves the better results for all the nontrivial benchmarks.

The improvements in execution times are less than the improvements in space, especially for smaller circuits. This is because separator based algorithms spend more time in the ordering phase itself. However, for larger circuits, this cost gets amortized by the smaller BDDs achieved during analysis. An important observation that can be made is that in general, our algorithms spend more time in the initial ordering phase as compared to FMCAD00. This is to be expected since both KLin and simulated annealing are optimization methods.

The last two columns in Table 2 indeed demonstrate that our algorithms improve various $\lambda_{\mathrm{s}}$ with respect to FMCAD'00. The main objective of our algorithms was to improve $\lambda_{L}$, though we can see that they also result in better $\lambda_{U} \mathrm{~s}$ in general.

\section{Conclusions and Future Work}

We have given convincing evidence that variable lifetimes have a crucial impact on the performance of image computation algorithms. We have also presented new algorithms for conjunction scheduling based on hill climbing, simulated annealing, and graph separators and shown the effectiveness of them. The per- 


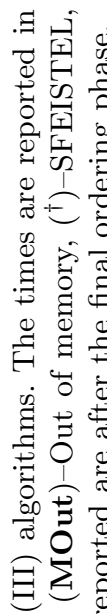

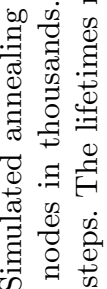

$\tau$

宅言

छ.

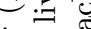

莙岩焉

ซำ

क्षे

焉些

.ี क्षे की

듀 ᄚ

वृ 2,

$.00 \%$

司

$\therefore \leqslant$

둬워

융

육

$\mathbb{S}_{0}$

$\mathcal{U} \cdot *$

$\sum_{1} \dot{U}^{-1}$

so 0

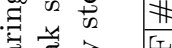

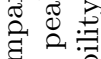

ฮै

$\circlearrowright$ E

ง $\dot{0}$

늘

E

F

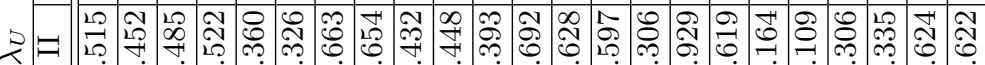

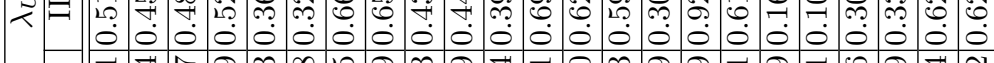

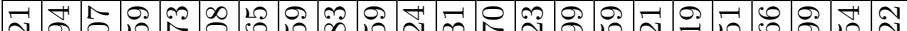

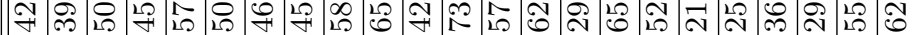
\begin{tabular}{lllllllllllllllllllllll}
$\dot{0}$ & 0 & 0 & 0 & 0 & 0 & 0 & 0 & 0 & 0 & 0 & 0 & 0 & 0 & 0 & 0 & 0 & 0 & 0 & 0 & 0 & 0 & 0 \\
\hline
\end{tabular}

-

$\exists$ F

m

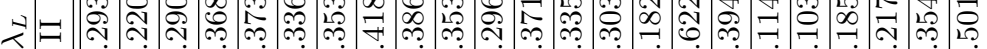
\begin{tabular}{lllllllllllllllllllllll}
0 & 0 & 0 & 0 & 0 & 0 & 0 & 0 & 0 & 0 & 0 & 0 & 0 & 0 & 0 & 0 & 0 & 0 & 0 & 0 & 0 & 0 & 0 \\
\hline
\end{tabular}

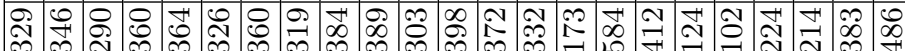

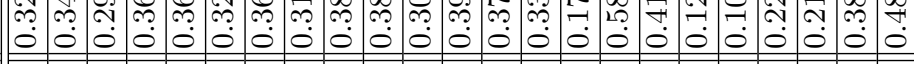

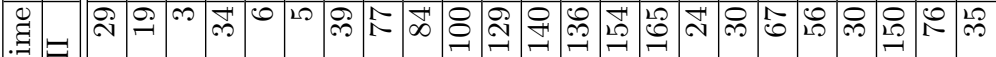

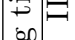

:

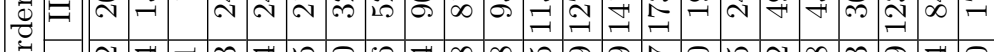

O-

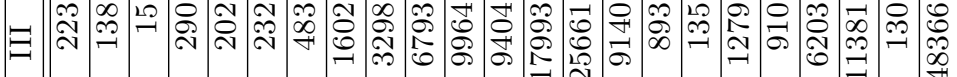

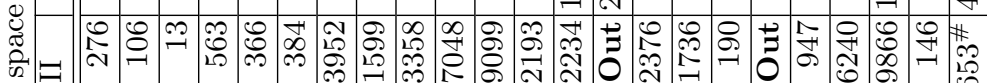

بै

ص

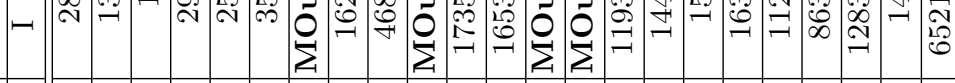

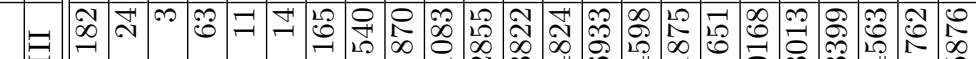

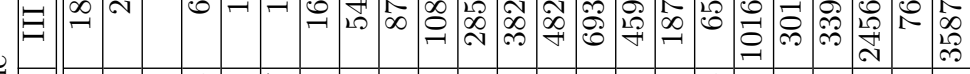

.

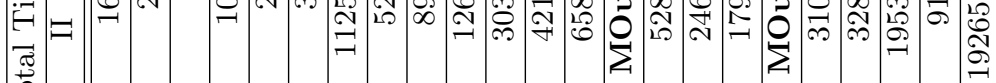

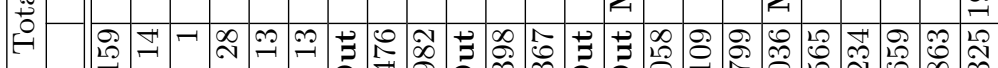

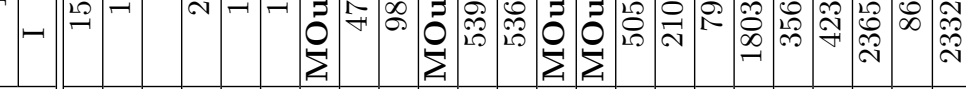

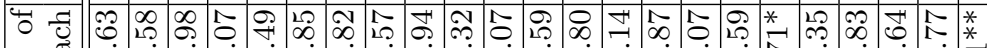

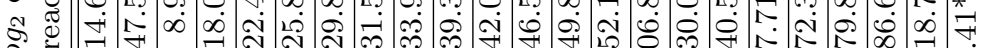

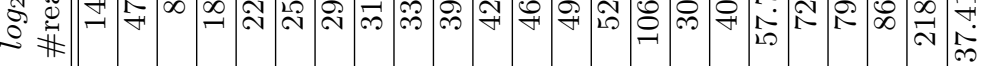

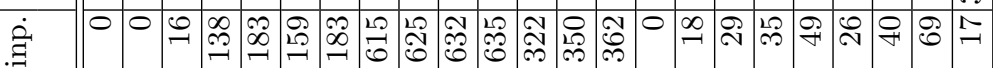

\#

在

\#

$\stackrel{\rightleftarrows}{\rightrightarrows}$

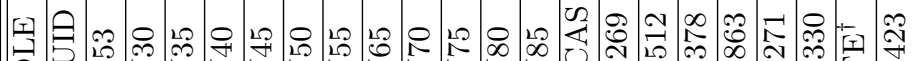
记 
formance of these algorithms was comparable to that of the current best known methods. Our experiments clearly demonstrate that for large circuits, we can achieve savings in memory in the range of $50-60 \%$. Since fine-tuned image computation algorithms are obviously most important for large circuits, we believe that our results are a significant contribution to model checking and reachability analysis. On the implementation side, we have contributed several new image computation algorithms to the NuSMV model checker, and believe that it will be a valuable research tool.

There are some interesting research directions to pursue. First of all, we need to understand the behavior of our algorithms on broader class of systems. The examples were mostly chosen from the circuit domain, and we would like to see the effectiveness of these algorithms on other class of circuits. Secondly, techniques which switch between different conjunction schedules depending on intermediate state sets in the fixpoint computation seem to be promising. We also plan to study in greater depth the effect of various parameters of our methods and automatic ways to tune them.

\section{References}

[BCL91a] J. R. Burch, E. M. Clarke, and D. E. Long. Representing circuits more efficiently in Symbolic Model Checking. In 28th ACM/IEEE Design Automation Conference, 1991.

[BCL91b] J. R. Burch, E. M. Clarke, and D. E. Long. Symbolic Model Checking with partitioned transition relations. In A. Halaas and P. B. Denyer, editors, Proceedings of the International Conference on Very Large Scale Int egration, Edinburgh, Scotland, August 1991.

[B'e92] C. J. P. B'elisle. Convergence theorems for a class of simulated annealing algorithms. Journal of Applied Probability, 29:885-892, 1992.

[BJ92] T. N. Bui and C. Jones. Finding good approximate vertex and edge partitions is NP-hard. Information Processing Letters, 42:153-159, 1992.

[CCGR99] A. Cimatti, E. M. Clarke, F. Giunchiglia, and M. Roveri. NuSMV: A new Symbolic Model Verifier. In N. Halbwachs and D. Peled, editors, Proceedings of International Conference on Computer-Aided Verification ( $\left.C A V^{\prime} 99\right)$, number 1633 in Lecture Notes in Computer Science, pages 495499. Springer, July 1999.

[CGP00] E. M. Clarke, O. Grumberg, and D. Peled. Model Checking. MIT Press, 2000 .

[FM82] C.M. Fiduccia and R.M. Mattheyses. A linear time heuristic for improving network partitions. In 19th ACM/IEEE Design Automation Conference, pages $175-181,1982$.

[GB94] D. Geist and I. Beer. Efficient Model Checking by automated ordering of transition relation partitions. In D. L. Dill, editor, Sixth Conference on Computer Aided Verification (CAV'94), volume 818 of LNCS, pages 299-310, Stanford, CA, USA, 1994. Springer-Verlag.

[GJ79] Michael R. Garey and David S. Johnson. Computers and Intractability: A Guide to the Theory of NP-Completeness. W. H. Freeman \& Co., 1979.

[Haj85] B. Hajek. A tutorial survey of theory and applications of simulated annealing. In Proc. 24th IEEE Conf. Decision and Control, pages 755-760, 1985 . 
[KJV83] S. Kirkpatrick, C. D. Gelatt Jr., and M. P. Vecchi. Optimization by simulated annealing. Science, 220:671-679, 1983.

[KL70] Brian Kernighan and S. Lin. An efficient heuristic procedure for partitioning graphs. The Bell System Technical Journal, pages 291-307, February 1970.

[MKRS00] In-Ho Moon, James H. Kukula, Kavita Ravi, and Fabio Somenzi. To split or to conjoin: The question in image computation. In Proceedings of the 37 th Design Automation Conference (DAC'00), pages 26-28, Los Angeles, June 2000.

$\left[\mathrm{MRR}^{+} 53\right]$ N. Metropolis, A. W. Rosenbluth, M. N. Rosenbluth, A. H. Teller, and E. Teller. Equation of state calculations by fast computing machines. Journal of Chemical Phyics, 21(6):1087-1092, 1953.

[MS00] In-Ho Moon and Fabio Somenzi. Border-block triangular form and conjunction schedule in image computation. In Warren A. Hunt Jr. and Steven D. Johnson, editors, Proceedings of the Formal Methods in Computer Aided Design (FMCAD'00), volume 1954 of LNCS, pages 73-90, November 2000.

$\left[\right.$ RAP $^{+}$95] R.K. Ranjan, A. Aziz, B. Plessier, C. Pixley, and R.K. Brayton. Efficient $\mathrm{BDD}$ algorithms for FSM synthesis and verification. In IEEE/ACM International Workshop on Logic Synthesis, Lake Tahoe, 1995. IEEE/ACM.

$\left[\mathrm{TSL}^{+}\right.$90] H. Touati, H. Savoj, B. Lin, R. K. Brayton, and A. Sangiovanni-Vincentelli. Implicit enumeration of finite state machines using BDDs. In Proceedings of the IEEE international Conference on Computer Aided Design (ICCAD), pages 130-133, November 1990.

[Yan99] Bwolen Yang. Optimizing Model Checking Based on BDD Characterization. PhD thesis, Carnegie Mellon University, Computer Science Department, May 1999.

\section{Appendix A}

It is easy to see that for a given permutation $\sigma$ of rows, we can compute $\lambda$ in polynomial time $(O(n \cdot m))$ and check if $\lambda \leq r$.

To show that $\lambda-O P T$ is NP-hard, we reduce a known NP-complete problem called optimal linear arrangement (OLA) [GJ79, page 200] to $\lambda-O P T$. An instance of OLA consists of a graph $G(V, E)$ and a positive integer $K$. The question is whether there exists a permutation $f$ of $V$ such that $\sum_{(u, v) \in E}|f(u)-f(v)| \leq K$. The reduction consists of constructing a dependence matrix $D$ and a number $r$ such that $(V, E), K$ is a solution of OLA iff $D, r$ is a solution to $\lambda-O P T$. An example of a reduction is given in figure 5 .

Formally, $D$ has $|V|$ rows corresponding to the vertices of $G(V, E)$, and $|E|$ columns corresponding to the edges of $G(V, E)$. For any edge $e_{k}=\left(v_{i}, v_{j}\right)$, set $d_{i k}=d_{j k}=1$ and set all other $d_{i j}$ 's to 0 . Thus, in each column there are two occurences of the symbol 1. We set $r=\frac{K+n}{n \cdot m}$. Trivially we obtain the following equivalence:

$$
\begin{aligned}
& \frac{\sum_{1 \leq j \leq n}\left(h_{j}-l_{j}+1\right)}{n \cdot m} \leq r \\
& \Leftrightarrow \sum_{1 \leq j \leq n}\left(h_{j}-l_{j}+1\right) \leq r \cdot(n \cdot m) \\
& \Leftrightarrow \sum_{1 \leq j \leq n}\left(h_{j}-l_{j}+1\right) \leq K+n
\end{aligned}
$$




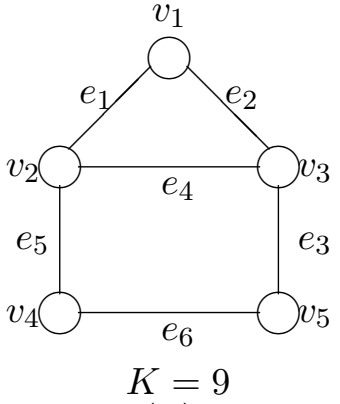

(a)

$$
\begin{aligned}
& \begin{array}{r|llll}
1 & 23456 \\
\hline 1 & 1 & 10000
\end{array} \\
& 2100110 \\
& 3011100 \\
& 40001001 \\
& 5 \mid \begin{array}{llllll}
0 & 0 & 0 & 0 & 1 & 1
\end{array} \\
& r=\frac{K+n}{n \cdot m}=1 / 2
\end{aligned}
$$

(b)

Fig. 5. (a) An instance of Optimal Linear Arrangement, (b) its reduction to $\lambda-O P T$. The permutation $v_{1}, v_{2}, v_{3}, v_{5}, v_{4}$ is a solution to both.

Let $\sigma$ be a permutation of the vertices of $V$. Note that $\sigma$ simultaneously is a permutation of the rows of $D$. We have to show that $\sigma$ is a solution of $G(V, E), K$ iff $\sigma$ is a solution of $D, r$.

The important observation is that because of the construction of $D$, the only nonzero entries in each column $j$ correspond to the two vertices of the edge $e_{j}=(u, v)$. Therefore, we conlude that $h_{j}-l_{j}=|\sigma(u)-\sigma(v)|$. Continuing the above equivalence we obtain

$$
\begin{aligned}
& \sum_{1 \leq j \leq n}|\sigma(u)-\sigma(v)|+n \leq K+n \\
& \Leftrightarrow \sum_{(u, v) \in E}|f(u)-f(v)| \leq K
\end{aligned}
$$

